\title{
Association testing by haplotype-sharing methods applicable to
} whole-genome analysis

\author{
Ilja M Nolte ${ }^{\dagger 1}$, André R de Vries ${ }^{\dagger 2}$, Geert T Spijker ${ }^{3}$, Ritsert C Jansen ${ }^{4}$, \\ Dumitru Brinza ${ }^{5}$, Alexander Zelikovsky ${ }^{5}$ and Gerard J te Meerman*2
}

\begin{abstract}
Address: ${ }^{1}$ Department of Epidemiology, University Medical Center Groningen, University of Groningen, Hanzeplein 1, 9713 GZ Groningen, the Netherlands, ${ }^{2}$ Department of Genetics, University Medical Center Groningen, University of Groningen, Hanzeplein 1, 9713 GZ Groningen, the Netherlands, ${ }^{3}$ Deparment of Dermatology, University Medical Center Groningen, University of Groningen, Hanzeplein 1, 9713 GZ Groningen, the Netherlands, ${ }^{4}$ Groningen Bioinformatics Center, Groningen Biomolecular Sciences and Biotechnology Institute, University of Groningen, Kerklaan 30, 9751 NN Haren, the Netherlands and ${ }^{5}$ Department of Computer Science, Georgia State University, 34 Peachtree Street, Atlanta, Georgia 30303-3086, USA
\end{abstract}

Email: Ilja M Nolte - i.m.nolte@med.umcg.nl; André R de Vries - andredevries@gmail.com; Geert T Spijker - g.t.spijker@derm.umcg.nl; Ritsert C Jansen - r.c.jansen@rug.nl; Dumitru Brinza - dima@cs.gsu.edu; Alexander Zelikovsky - alexz@cs.gsu.edu; Gerard J te Meerman* - g.j.te.meerman@med.umcg.nl

* Corresponding author †Equal contributors

from Genetic Analysis Workshop 15

St. Pete Beach, Florida, USA. II-I5 November 2006

Published: 18 December 2007

BMC Proceedings 2007, I (SuppI I):SI 29

This article is available from: http://www.biomedcentral.com/I753-656I/I/SI/SI29

(c) 2007 Nolte et al; licensee BioMed Central Ltd.

This is an open access article distributed under the terms of the Creative Commons Attribution License (http://creativecommons.org/licenses/by/2.0), which permits unrestricted use, distribution, and reproduction in any medium, provided the original work is properly cited.

\begin{abstract}
We propose two new haplotype-sharing methods for identifying disease loci: the haplotype sharing statistic (HSS), which compares length of shared haplotypes between cases and controls, and the CROSS test, which tests whether a case and a control haplotype show less sharing than two random haplotypes. The significance of the HSS is determined using a variance estimate from the theory of U-statistics, whereas the significance of the CROSS test is estimated from a sequential randomization procedure. Both methods are fast and hence practical, even for whole-genome screens with high marker densities. We analyzed data sets of Problems 2 and 3 of Genetic Analysis Workshop 15 and compared HSS and CROSS to conventional association methods. Problem 2 provided a data set of 2300 single-nucleotide polymorphisms (SNPs) in a 10-Mb region of chromosome 18q, which had shown linkage evidence for rheumatoid arthritis. The CROSS test detected a significant association at approximately position $4407 \mathrm{~kb}$. This was supported by singlemarker association and HSS. The CROSS test outperformed them both with respect to significance level and signal-to-noise ratio. A $20-\mathrm{kb}$ candidate region could be identified. Problem 3 provided a simulated $10 \mathrm{k}$ SNP data set covering the whole genome. Three known candidate regions for rheumatoid arthritis were detected. Again, the CROSS test gave the most significant results. Furthermore, both the HSS and the CROSS showed better fine-mapping accuracy than straightforward haplotype association. In conclusion, haplotype sharing methods, particularly the CROSS test, show great promise for identifying disease gene loci.
\end{abstract}




\section{Background}

With the current advances in genotyping technology, genome-wide association studies for detecting genes involved in complex diseases have recently become feasible. However, the computational and statistical methodology for analyzing such studies needs optimization and standardization. Various methods and strategies have been investigated [1-3]. The typical association study of candidate regions uses single locus tests, such as the chisquare test or the transmission disequilibrium test [4]. The advantage of single-locus tests is that haplotype inference can be avoided. However, it is expected that there is more information contained in haplotypes as a result of the underlying evolutionary processes $[5,6]$. Therefore, association analysis based on haplotypes is believed to be more powerful, in particular if the common disease-common variant theory applies. According to this, the genetic variants related to a complex disease are old mutations and are common in the population (minor allele frequencies $>5 \%$ ). Subsequent mutations and recombinations in the ancestral haplotype at which the disease mutation occurred shortened the haplotypes that descended from this ancestor. However, in the current generation the haplotypes will still share a fragment around the disease locus. The size of the reduced ancestral haplotype fragments varies as a result of the uneven nature of such recombination and mutation processes. We hypothesize that there is a difference in haplotype patterns between cases and controls at regions associated with the disease and we present two new methods based on this hypothesis: the haplotype-sharing statistic (HSS), which is an improvement of a statistic described by Van der Meulen et al. $[7,8]$, and the CROSS test, which was published in an earlier form [9]. The test statistics of these two haplotypesharing methods will be described and their performance will be compared to standard association methods.

\section{Methods \\ Materials}

Data from both Problems 2 and 3 of Genetic Analysis Workshop 15 (GAW15) were analyzed. From Problem 2, we used a data set of the North American Rheumatoid Arthritis Consortium (NARAC) of 2300 single-nucleotide polymorphisms (SNPs) covering $10 \mathrm{Mb}$ of chromosome $18 \mathrm{q}$, a region that had shown linkage evidence for rheumatoid arthritis (RA) [10]. This data set contained 460 cases and 460 controls, the latter being recruited from a New York City population. Problem 3 provided simulated RA data on 9187 SNPs distributed over the entire genome. We used Replicates 1 through 10 of the affected sib-pair nuclear families for analysis. From each family, haplotypes transmitted to the first affected sib were used as cases and non-transmitted haplotypes as controls, regardless of the affection status of the parents. We had no prior knowledge of the answers.

\section{Haplotype inference}

The 920 subjects of the NARAC data set were phased using the phasing program 2SNP [11]. This program reconstructed the 1840 haplotypes for all 2300 SNPs in approximately 20 minutes. The construction of the simulated data sets allowed extraction of phased haplotypes and these data sets were analyzed without phase ambiguities or missing alleles.

\section{Statistical analysis}

Haplotype sharing between two haplotypes $X$ and $Y$ from the perspective of each locus $k$, denoted as $h(X, Y ; k)$, can be evaluated as the number of consecutive SNPs in the telomeric and centromeric directions carrying the same alleles including locus $k$. Given a sample of case haplotypes $X_{1}, \ldots, X_{N}$ and a sample of control haplotypes $Y_{1}, \ldots, Y_{M}$, four measures of haplotype sharing at locus $k$ are defined as follows:

case sharing:

$\operatorname{SH}^{\text {CASE }}(k)=\frac{2}{N(N-1)} \cdot \sum_{i=1}^{N-1} \sum_{j=i+1}^{N} h\left(X_{i}, X_{j} ; k\right) ;$

control sharing:

$S H^{C T R}(k)=\frac{2}{M(M-1)} \cdot \sum_{i=1}^{M-1} \sum_{j=i+1}^{M} h\left(Y_{i}, Y_{j} ; k\right)$;

cross sharing: $\operatorname{SH}^{\text {CROSS }}(k)=\frac{1}{M N} \cdot \sum_{i=1}^{N} \sum_{j=1}^{M} h\left(X_{i}, Y_{j} ; k\right)$;

overall sharing:

$S H^{A L L}(k)=\frac{N^{2} \cdot S H^{C A S E}(k)+M^{2} \cdot S H^{C T R}(k)+2 N M \cdot S H^{C R O S S}(k)}{(N+M)^{2}}$

The first haplotype sharing method, the HSS, compares the case haplotype sharing with control haplotype sharing. In contrast to the earlier HSS $[7,8]$, in this manner the HSS corrects for linkage disequilibrium (LD) other than that caused by the disease mutation. We hypothesize that haplotype sharing will be larger among cases than among controls at loci involved in the disease and at other loci in LD with them, because i) haplotypes containing a risk allele are more likely to be similar to each other and dissimilar to haplotypes containing a non-risk allele; and ii) haplotypes containing the risk allele may be shared over longer stretches. The first factor is explained from the concepts of association and LD. The second factor can be explained by presumably shorter coalescence times of disease alleles and hence fewer recombination events in the 
sample of cases compared to the sample of wild-type alleles in controls. The HSS at locus $k$ is defined as

$$
t_{H S S}(k)=\frac{S H^{C A S E}(k)-S H^{C T R}(k)}{\sqrt{\left(s d S H^{C A S E}(k)\right)^{2}+\left(s d S H^{C T R}(k)\right)^{2}}},
$$

where $s d S H^{C A S E}(k)$ and $s d S H^{C T R}(k)$ are the estimates of the standard deviation of the mean haplotype sharing at locus $k$ accounting for LD among cases and controls, respectively. When $N$ and $M$ are large, $\operatorname{SHCASE}(k)$ and $S H^{C T R}(k)$ follow a normal distribution (Central Limit Theory) and, because $S H^{C A S E}(k)$ and $S H^{C T R}(k)$ are independent, significances of $t_{H S S}(k)$ can be derived from a $t$-distribution with $N+M-2$ degrees of freedom (i.e., $N-1$ from the cases and $M-1$ from the controls). The main statistical problem in evaluating mean haplotype sharing is how to calculate the variance of the mean sharing between all pairs of haplotypes. Generally, haplotypes will share alleles in groups and this means that haplotype agreement between haplotype pairs is not independent. For the HSS, we derived an unbiased estimate from the theory of U-statistics for the standard deviation of the mean haplotype sharing (see Appendix).

The second haplotype-sharing method is the CROSS test. This hypothesizes that a case and a control haplotype are different from each other in the region of a disease locus and will therefore show less haplotype sharing (cross sharing; $\left.S H^{C R O S S}\right)$ than two random haplotypes $\left(S H^{A L L}\right)$. This test incorporates more information on allele frequency differences between cases and controls (i.e., the single SNP association "signal") than the HSS. Unlike the HSS, an equivalent U-statistics variance of the cross sharing can not be estimated because of the correlation between $S H^{C R O S S}$ and $S H^{A L L}$. Therefore, the variance of the cross sharing is estimated from a sequential randomization procedure in which case and control status is randomly permuted over the haplotypes as long as the interim significance estimate remains interesting (i.e., $p$ value $<0.1$ ). In order to render this test fast and hence feasible for whole-genome screens with a high density of SNPs, the significance is not determined from the randomization procedure, but the variance of $\operatorname{SHCROSS}^{(k)}$ $S H^{A L L}(k)$ is estimated from a maximum of 200 randomizations, which is a sufficient number to provide a reasonably accurate variance estimate. The CROSS test at locus $k$ is then defined as

$$
z_{\text {CROSS }}(k)=\frac{S H^{C R O S S}(k)-S H^{A L L}(k)}{s d\left(S H^{C R O S S}(k)-S H^{A L L}(k)\right)} .
$$

Note that a negative value implicates positive association. As a result of the correlation between $S H^{C R O S S}$ and $S H^{A L L}$, the tails of the $z_{\text {CROSS }}(k)$ distribution are not properly approximated by a normal distribution, leading to downward biased $p$-values for extreme $z$-values. Therefore, the $z$ values are transformed to a chi-square distribution with $v$ degrees of freedom:

$$
P\left(\chi^{2} \mid v\right) \sim \Phi(\mathrm{z}) \text { where } \mathrm{z}=\frac{\chi^{2}-v}{\sqrt{2 v}} \Rightarrow \chi_{\text {CROSS }}^{2}(\mathrm{k}) \sim \mathrm{z}_{\mathrm{CROSS}}(\mathrm{k}) \cdot \sqrt{2 v}+v .
$$

With an appropriately chosen $v$, the distribution resembles the true $z$-score distribution, especially in the tails, so that realistic $p$-values are obtained. The best choice for $v$ typically depends on the sample size and on the individual chromosome. For the current study, we empirically derived the value for $v$ that minimized the bias in $p$-values in non-associated regions.

In order to compare the performances of the HSS and CROSS, we also performed single-SNP and haplotypeassociation analysis. Single-SNP association was tested by means of a chi-square test. For haplotype association, frequencies of haplotypes of five consecutive SNPs were counted and a log-likelihood ratio test was performed including only haplotypes with $n>10$ to assess the significance of the difference between cases and controls (our own software, available on request).

We used a conservative Bonferroni correction to correct for multiple testing. Hence, in the real data, a result was considered significant if the $-\log (p$-value) was larger than $-\log (0.05 / 2300)=4.65$, and in the simulation study if the $-\log (p$-value $)$ was larger than $-\log (0.05 / 9187)=5.25$ and suggestive if it was larger than $-\log (0.10 / 9187)=4.95$.

\section{Results \\ NARAC data set (Problem 2)}

The running time of all analyses for 2300 SNPs and 920 individuals was 80 minutes on a single laptop PC (Celeron $1500 \mathrm{MHz}$ ), which is sufficiently fast to be acceptable for whole-genome association studies.

The results are presented in Figures 1 and 2. The CROSS test revealed a significant association with a $-\log (p$-value) of 6.6 at position $4407 \mathrm{~kb}$ from the first SNP. The odds ratio based on allele frequencies at this location is 1.41 (1.16-1.72, 95\% CI). A second, non-significant peak ($\log (p$-value $)=4.1)$ was found near $4863 \mathrm{~kb}$ from the first SNP, with a corresponding odds ratio of 1.21 (0.99-1.51, $95 \% \mathrm{CI})$. Although the HSS peaks at the same two locations $(-\log (p)=4.2$ and 3.2, respectively), these results were not significant. The same holds for the single-marker chi-square test.

Figure 2 focuses on the associated region around $4407 \mathrm{~kb}$. The CROSS test did not show a clear peak at a particular locus. Instead, several SNPs in the region were associated, 


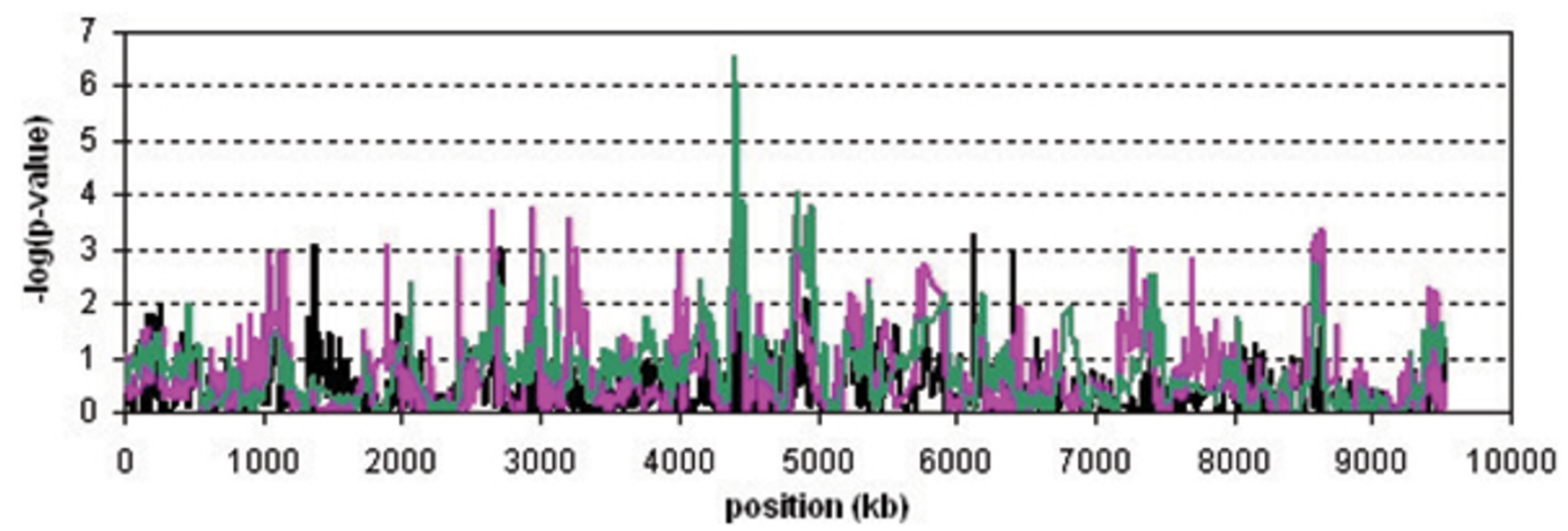

Figure I

Analysis results of the NARAC data set. Results of the CROSS test (green line), the HSS (pink line) and single-marker association (black line) are plotted for all 2300 SNPs on chromosome 18q.

which was to be expected because the CROSS test is based on haplotype sharing and tests at subsequent loci were highly correlated. However, the HSS and the singlemarker chi-square test were not informative about the exact disease locus either. Nevertheless, the CROSS identified a small region of $20 \mathrm{~kb}(4,400-4,420 \mathrm{~kb})$ as the candidate region.

\section{Simulated data set (Problem 3)}

Figure 3 shows the results of the genome-wide SNP association analyses. Genome-wide significant associations were observed for all statistical methods at chromosomal regions $6 \mathrm{p} 21$ and $11 \mathrm{q} 23.1$, and we found a suggestive result at $18 \mathrm{q} 22.2$ for the CROSS test only. These regions are known candidate regions for rheumatoid arthritis. The CROSS test showed the most significant results: for $6 \mathrm{p} 21$ the mean of the $-\log (p)$-values for the ten replicates was 443.6 (range 370.3-552.0); for $11 \mathrm{q} 23.1$ it was 22.8 (range 6.4-33.9); and for 18q22.2 it was 4.95 (range 1.810.6). None of the methods detected other associated regions.

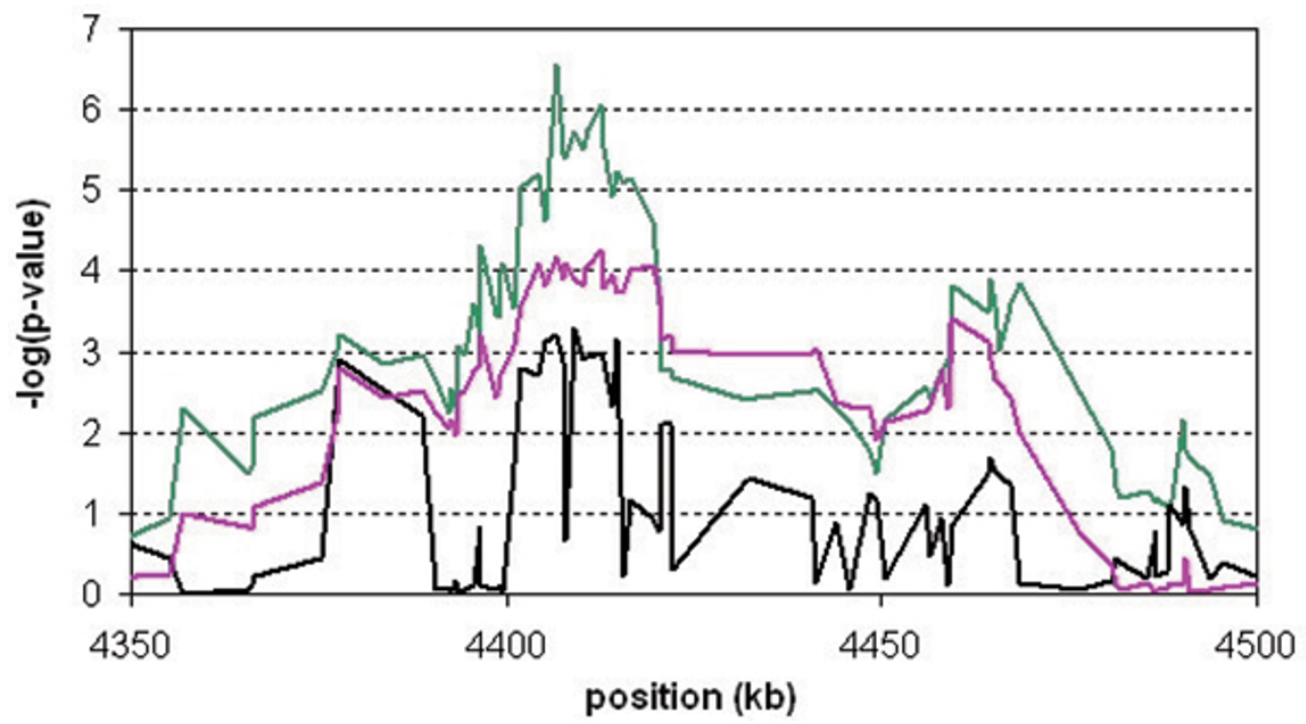

Figure 2

Region showing the strongest association in the NARAC data set. The CROSS (green line), the HSS (pink line) and single-SNP association (black line) - $\log (p$-values) are plotted for the I50-kb region showing the strongest association. 


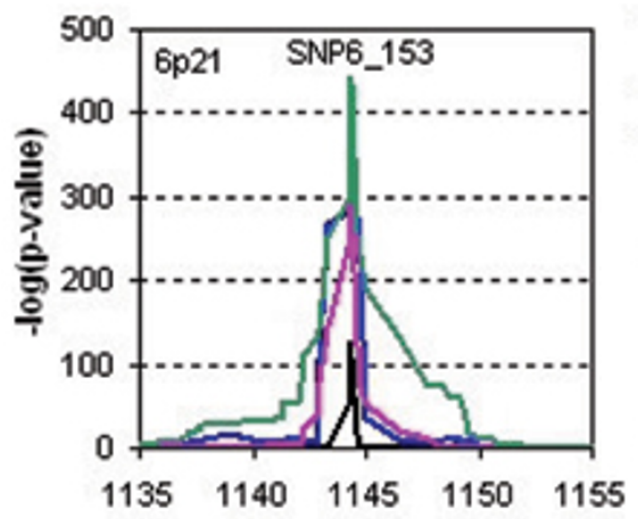

Figure 3

Analysis results of the simulated data set in the regions 6p2 I (a), I Iq23.I (b), and I8q22.2 (c). On the x-axis, the cumulative position with respect to the start of chromosome I is given. The black, blue, pink, and green lines represent the mean of the $-\log (p$-values) of the ten replicates of single-SNP association, five-SNP haplotype association, HSS, and CROSS, respectively.

Type I errors and power are shown in Table 1. Both haplotype-sharing methods had the correct type I errors. On chromosome 11 both the HSS and the CROSS tests had more power than the standard association analyses, while on chromosome 18 only the CROSS test had more power. The significance of the effect on chromosome 6 was so strong that all methods had $100 \%$ power in this region.

\section{Discussion}

The results of our analyses on both the NARAC data and simulated genome-wide data show that the CROSS test is a powerful statistic for identifying disease loci. The CROSS combines information on differences in haplotype sharing, which are also used by the HSS, and on allele frequency differences, which are the basis of association analysis.

In the NARAC data, the CROSS test revealed a significant association $(-\log (p$-value $)=6.6)$ approximately $4407 \mathrm{~kb}$ from the starting SNP. Odds ratios of 1.41 were observed at multiple SNPs in this region. A second suggestive peak was observed near $4863 \mathrm{~kb}(-\log (p$-value $)=4.1)$. This result was not significant after applying the conservative Bonferroni multiple testing correction, but because the significances outside of the associated region seemed to range up to a $-\log (p)$ of $\sim 3$, this result might indicate a relevant association as well. The HSS and the single-SNP association test peaked at the same locations as the CROSS, but these tests did not reach significance. Furthermore, the HSS and single-SNP association analyses were not specific enough because multiple signals at other positions reached the same significance level as the $4407 \mathrm{~kb}$ peak. The CROSS test did not identify a particular SNP as the causal one, which is inherent to the way the test was developed, but a candidate region as small as $20 \mathrm{~kb}$ could clearly be identified.

Table I: Type I error and power

\begin{tabular}{|c|c|c|c|c|c|c|}
\hline \multirow[b]{2}{*}{ Method } & \multicolumn{3}{|c|}{ Type I error ${ }^{\mathrm{a}}$} & \multicolumn{3}{|c|}{ Powerb } \\
\hline & 0.05 & 0.005 & 0.0005 & Chr 6 & Chr II & Chr 18 \\
\hline Single-SNP association & 0.051 & 0.0048 & 0.00057 & 1.0 & 0.7 & 0.1 \\
\hline Haplotype association & 0.053 & 0.0052 & 0.00051 & 1.0 & 0.8 & 0.1 \\
\hline HSS & 0.052 & 0.0046 & 0.00031 & 1.0 & 1.0 & 0.1 \\
\hline CROSS & 0.051 & 0.0051 & 0.00061 & 1.0 & 1.0 & 0.4 \\
\hline
\end{tabular}

aType I results at three different significance levels were determined from the Replicates I to 10 of SNPs on all chromosomes except 6 , II, and I8. bPower is determined as the fraction of replicates showing a significant result ( $P<0.05 / 9187$ ) in a I-Mb region around SNP6_I53 on chromosome 6, SNPII_389 on chromosome II, and SNPI8_269 on chromosome 18. 
When applied to the simulated $10 \mathrm{k}$ SNP array data, the HSS and CROSS tests showed significant results in the known candidate regions for rheumatoid arthritis, i.e., 6p21 and 11q23.1, and the CROSS was suggestive of association at 18q22.2 after Bonferroni correction. The type I errors of these haplotype-sharing methods were as expected and no significant associations in other regions were observed. Similar results were found by single-SNP and five-SNP haplotype association analysis, but the CROSS test showed more power in each associated region.

The strength of single-SNP association analysis was inferior to that of our two haplotype sharing methods, suggesting that the latter indeed contain more information. The HSS and CROSS showed more significant results and sharper peaks than the five-SNP haplotype analysis. This might imply that HSS and CROSS have a better mapping accuracy than a fixed-length or sliding-window haplotype method. This could result from the fact that HSS and CROSS use the variable length over which haplotypes are the same, whereas a sliding window method may use either too much or too little information. The finding that both the HSS and the CROSS test accurately identify the disease locus might seem to conflict with the results of the NARAC data, in which the exact locus could not be pinpointed. However, the SNP density of the NARAC data is about 80 -fold higher than in the simulated data set and multiple highly correlated SNPs were expected to be associated causing broader peaks.

We did not investigate the robustness of the haplotype sharing methods to haplotype reconstruction errors or missing alleles. Although this analysis would be interesting and remains necessary, the focus of this paper was to introduce and describe the HSS and the CROSS test. The effects of phase ambiguities and missing alleles on the HSS and CROSS will be the topic of future work.

\section{Conclusion}

The HSS and in particular the CROSS test show great promise for identifying and fine-mapping disease genes of complex diseases. They are useful for whole genome association screens because the analytical form of the HSS and the sequential randomization for estimating variance of the CROSS test renders them fast enough and hence practical to use even for marker densities of 500,000 SNPs/ genome.

\section{Appendix}

The mean haplotype sharing is a U-statistic: $U(k)=\frac{2}{N(N-1)} \cdot \sum_{i=1}^{N-1} \sum_{j=i+1}^{N} h\left(X_{i}, X_{j} ; k\right)$. Denote the mean and variance of the sharing of two haplotypes at locus $k$,
$h\left(X_{i}, X_{j} ; k\right)$, by $\mu_{k}$ and $\sigma_{k}^{2}$, respectively. We state that, given a data set $\left\{X_{1}, \ldots, X_{N}\right\}$,

$S(k)^{2}=S_{h}(k)^{2}-\frac{2}{N(N-1)} \cdot \sum_{i=1}^{N-1} \sum_{j=i+1}^{N}\left(h\left(X_{i}, X_{j} ; k\right)\right)^{2}+U(k)^{2}$,

where

$$
\begin{aligned}
S_{h}(k)^{2} & =\frac{1}{(N-2)(N-3)} \cdot\left\{((N-2)(N-3)-2) \cdot\left(\frac{2}{N(N-1)} \sum_{i=1}^{N-1} \sum_{j=i+1}^{N}\left(h\left(X_{i}, X_{j} ; k\right)\right)^{2}\right)\right. \\
& \left.+4(N-1) \cdot \frac{1}{N} \sum_{i=1}^{N}\left(\frac{1}{N-1} \sum_{\substack{j=1 \\
j \neq i}}^{N} h\left(X_{i}, X_{j} ; k\right)\right)^{2}-N(N-1) \cdot U(k)^{2}\right\},
\end{aligned}
$$

is an unbiased estimate of the variance of a U-statistic. We will derive that $S_{h}(k)^{2}$ is an unbiased estimate of $\operatorname{var}\left(h\left(X_{i^{\prime}}\right.\right.$ $\left.\left.X_{j} ; k\right)\right)=\sigma_{k}^{2}$, because from this finding, it simply follows that $S(k)^{2}$ is an unbiased estimator of $\operatorname{var}(\mathrm{U})$, as

$$
\begin{aligned}
E\left(S(k)^{2}\right) & =E\left(S_{h}(k)^{2}\right)-\frac{2}{N(N-1)} \cdot \sum_{i=1}^{N-1} \sum_{j=i+1}^{N} E\left(h\left(X_{i}, X_{j} ; k\right)\right)^{2}+E\left(U(k)^{2}\right) \\
& =\sigma_{k}^{2}-\left(\sigma_{k}^{2}+\mu_{k}^{2}\right)+\left(\operatorname{var}(U(k))+\mu_{k}^{2}\right) \\
& =\operatorname{var}(U(k)) .
\end{aligned}
$$

It is simple to derive that

$\frac{2}{N(N-1)} \cdot \sum_{i=1}^{N-1} \sum_{j=i+1}^{N}\left(h\left(X_{i}, X_{j} ; k\right)\right)^{2}-\frac{4}{N(N-1)(N-2)(N-3)} \cdot \sum_{i=1}^{N} \sum_{j=i+1}^{N} \sum_{l=1}^{N} \sum_{\substack{l \\ l \neq i, j \\ m \neq 1+1}}^{N} h\left(X_{i}, X_{j} ; k\right) \cdot h\left(X_{l}, X_{m} ; k\right)$

is an unbiased estimator of $\sigma_{k}^{2}$ (proof left to the reader).

This estimator is of the order 4 , as in the second term each haplotype pair is considered twice. To speed up the calculation, this estimator can be rewritten to an estimator of the order 2 as follows:

$$
\begin{aligned}
& \frac{2}{N(N-1)} \sum_{i=1}^{N-1} \sum_{j=i+1}^{N}\left(h\left(X_{i}, X_{j} ; k\right)\right)^{2}-\frac{4}{N(N-1)(N-2)(N-3)} \cdot \sum_{i=1}^{N} \sum_{j=i+1}^{N} \sum_{\substack{l \\
l \neq i, j}}^{N} \sum_{\substack{m=1+1 \\
m \neq i, j}}^{N} h\left(X_{i}, X_{j} ; k\right) \cdot h\left(X_{l}, X_{m} ; k\right) \\
& =\frac{2}{N(N-1)} \cdot \sum_{i=1}^{N-1} \sum_{j=i+1}^{N}\left(h\left(X_{i}, X_{j} ; k\right)\right)^{2}-\frac{4}{N(N-1)(N-2)(N-3)} \cdot\left[\left(\sum_{i=1}^{N} \sum_{j=i+1}^{N} h\left(X_{i}, X_{j} ; k\right)\right)^{2}+\sum_{i=1}^{N} \sum_{j=i+1}^{N}\left(h\left(X_{i}, X_{j} ; k\right)\right)^{2}\right. \\
& -\sum_{i=1}^{N} \sum_{j=i+1}^{N} \sum_{m=i+1}^{N} h\left(X_{i}, X_{j} ; k\right) \cdot h\left(X_{i}, X_{m} ; k\right)-\sum_{i=1}^{N} \sum_{j=i+1}^{N} \sum_{m=j+1}^{N} h\left(X_{i}, X_{j} ; k\right) \cdot h\left(X_{j}, X_{m} ; k\right) \\
& \left.-\sum_{i=1}^{N} \sum_{j=i+1}^{N} \sum_{l=1}^{i-1} h\left(X_{i}, X_{j} ; k\right) \cdot h\left(X_{l}, X_{i} ; k\right)-\sum_{i=1}^{N} \sum_{j=i+1}^{N} \sum_{l=1}^{j-1} h\left(X_{i}, X_{j} ; k\right) \cdot h\left(X_{l}, X_{j} ; k\right)\right] \\
& =\left(1-\frac{2}{(N-2)(N-3)}\right) \cdot \frac{2}{N(N-1)} \cdot \sum_{i=1}^{N-1} \sum_{j=i+1}^{N}\left(h\left(X_{i}, X_{j} ; k\right)\right)^{2}-\frac{N(N-1)}{(N-2)(N-3)} \cdot\left(\frac{2}{N(N-1)} \sum_{i=1}^{N} \sum_{j=i+1}^{N} h\left(X_{i}, X_{j} ; k\right)\right)^{2}
\end{aligned}
$$

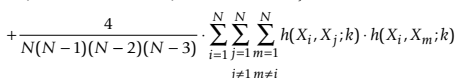

$$
\begin{aligned}
& =\frac{1}{(N-2)(N-3)} \cdot\left\{((N-2)(N-3)-2) \cdot \frac{2}{N(N-1)} \cdot \sum_{i=1}^{N-1} \sum_{j=i+1}^{N}\left(h\left(X_{i}, X_{j} ; k\right)\right)^{2}-N(N-1) \cdot U(k)^{2}\right. \\
& \left.+4(N-1) \cdot \frac{1}{N} \sum_{i=1}^{N}\left(\frac{1}{N-1} \sum_{\substack{j=1 \\
j \neq i}}^{N} h\left(X_{i}, X_{j} ; k\right)\right)^{2}\right\} \\
& =S_{h}^{2}(k) \text {. }
\end{aligned}
$$

\section{Competing interests}

The author(s) declare that they have no competing interests. 


\section{Acknowledgements}

This article has been published as part of BMC Proceedings Volume I Supplement I, 2007: Genetic Analysis Workshop 15: Gene Expression Analysis and Approaches to Detecting Multiple Functional Loci. The full contents of the supplement are available online at http://www.biomedcentral.com/

1753-6561/I?issue=SI.

\section{References}

I. Hirschhorn J, Daly M: Genome-wide association studies for common diseases and complex traits. Nat Rev Genet 2005, 6:95-108.

2. Gordon D, Finch S: Factors affecting statistical power in the detection of genetic association. J Clin Invest 2005, I I5:1408-1418.

3. Marchini J, Donelly P, Cardon L: Genome-wide strategies for detecting multiple loci that influence complex disease. Nat Genet 2005, 37:413-417.

4. Spielman R, Ewens W: The TDT and other family-based tests for linkage disequilibrium and association. Am J Hum Genet 1996, 59:983-989.

5. te Meerman G, Van der Meulen M, Sandkuijl L: Perspectives of identity by descent (IBD) mapping in founder populations. Clin Exp Allergy 1995, 25:97-102.

6. te Meerman G, Van der Meulen M: Genomic sharing surrounding alleles identical by descent: effects of genetic drift and population growth. Genet Epidemiol 1997, 14: I 225-I I 30.

7. Van der Meulen M, te Meerman G: Association and haplotype sharing due to identity by descent, with an application to genetic mapping. In Genetic Mapping of Disease Genes Edited by: Pawlowitzki I, Edwards J, Thompson E. London: Academic Press; 1997: I I5-135.

8. Van der Meulen M, te Meerman G: Haplotype sharing analysis in affected individuals from nuclear families with at least one affected offspring. Genet Epidemiol 1997, 14:915-920.

9. Levinson D, Kirby A, Slepner S, Nolte I, Spijker G, te Meerman G: Simulation studies of detection of a complex disease in a partially isolated population. Am J Med Genet 200I, I 05:65-70.

10. Jawaheer D, Seldin MF, Amos Cl, Chen WV, Shigeta R, Etzel C, Damle A, Xiao X, Chen D, Lum RF, Monteiro J, Kern M, Criswell LA, Albani S, Nelson JL, Clegg DO, Pope R, Schroeder HW, Bridges SL, Pisetsky DS, Ward R, Kastner DL, Wilder RL, Pincus T, Callahan LF, Flemming D, Wener MH, Gregersen PK, North American Rheumatoid Arthritis Consortium: Screening the genome for rheumatoid arthritis susceptibility genes: a replication study and combined analysis of 5 I 2 multicase families. Arthritis Rheum 2003, 48:906-916.

II. Brinza D, Zelikovsky A: 2SNP: Scalable phasing based on 2-SNP haplotypes. Biolnformatics 2006, 22:37I-373.

\section{Publish with Bio Med Central and every scientist can read your work free of charge}

"BioMed Central will be the most significant development for disseminating the results of biomedical research in our lifetime. "

Sir Paul Nurse, Cancer Research UK

Your research papers will be:

- available free of charge to the entire biomedical community

- peer reviewed and published immediately upon acceptance

- cited in PubMed and archived on PubMed Central

- yours - you keep the copyright

Submit your manuscript here:

http://www.biomedcentral.com/info/publishing_adv.asp
BioMedcentral 